\title{
Root Water Uptake Process for Different Types of Soil in Unsteady Infiltration from Periodic Trapezoidal Channels
}

\author{
Millatuz Zahroh ${ }^{1, *}$, Imam Solekhudin ${ }^{2}$ \\ ${ }^{1}$ Mathematics Department, Universitas Jember \\ ${ }^{2}$ Mathematics Department, Universitas Gadjah Mada \\ *Corresponding author.Email: millatuz@unej.ac.id
}

\begin{abstract}
This study involves a non-linear partial differential equation known as Richard's Equation. An unsteady infiltration from trapezoidal periodic irrigation channel with root-water uptake is considered as the problem. To solve the problem, A set of transformations, Kirchhoff transformation, dimensionless variables, Batu's transformation and Laplace transformation, are employed to transform Richard's Equation into a modified Helmholtz equation. Finally, the transformation is solved numerically using The Dual Reciprocity Method (DRM) with a predictor-corrector scheme. Employing Gaver-Stehfest formulae and diffusivity factor, distributions of root water uptake process are obtained as sink term of the problem.
\end{abstract}

Keywords: Root water uptake, Unsteady infiltration, DRM, Diffusivity factor.

\section{INTRODUCTION}

The problems of unsteady water infiltration from surface irrigation channels were studied by numerous researchers. Basha [1] studied multidimensional linearized nonsteady infiltration with prescribed boundary conditions at the soil surface. Barry and Parlange [2] considered Infiltration subject to timedependent surface ponding. Clements and Lobo [3] examined A BEM for time-dependent infiltration from an irrigation channel. In the previous reasearch, Zahroh and Sholekhudin [4] have considered solution time dependent infiltration at actual times by determining soil water diffusivity according to Chaudhari and Somawanshi [5]. However, the water absorption of plant roots or root water uptake process including unsteady infiltration was not taken under consideration.

The present paper employs solutions to the problem of the root water uptake process from different types of homogeneous soil in unsteady infiltration. A mathematical model to govern the problem is a Richard's Equation. By using a set of transformations including Laplace Transform, the governed equation is transformed into a modified Helmholtz Equation which is solved numerically by employing Dual
Reciprocity Method (DRM) with a predictor-corrector scheme simultaneously. Finally, the numerical solutions are obtained by Gaver-Stehfest formulae and diffusivity factor, until reaching the steady condition in actual times. The numerical solutions are then applied to examine the root water uptake process.

\section{METHOD}

The homogeneous soils considered in this study are clay-loam, clay soils, and silt-loam with trapezoidal channel irrigation furrows. The channels are assumed identical and sufficiently long which are kept full filled with water. It is also assumed no flux from the outside of the surface channel. A row of crops is planted equidistance between two contiguous channels. From the situations, the processes of root water uptake from unsteady infiltration are observed.

\subsection{Model Formulation}

Referred cartesian system $O X Y Z$ consider the homogeneous soil lying in $Z>0$ region as the positive direction. Since it was assumed $O Y-$ direction does not vary in geometrical shapes, the infiltration process may be assumed as two-dimensional flow. Besides that, Figure 1 of the problem described the plane $X=$ 
$\pm 100 k$ for $k=0,1,2, \ldots$ is symmetrical. Hence, region $R$ bounded by semi-infinite boundary $\mathrm{C}$ such $0 \leq X \leq 100 \mathrm{~cm}$ and $Z \geq 0$ is sufficiently considered as the domain of the problem, Figure 1 The distance between the center of a channel and the closest crops is $L+D$. Following Batu [7], we assume $\lim _{X \rightarrow \infty} \frac{\partial \theta}{\partial X}=0$ and $\lim _{Z \rightarrow \infty} \frac{\partial \theta}{\partial Z}=0$.For the root zone of crops, the transpiration process, the maximum rooting depth, and the maximum rooting width are respectively denoted by $2 \mathrm{Lt}, \mathrm{Zm}$ and $2 X m$.

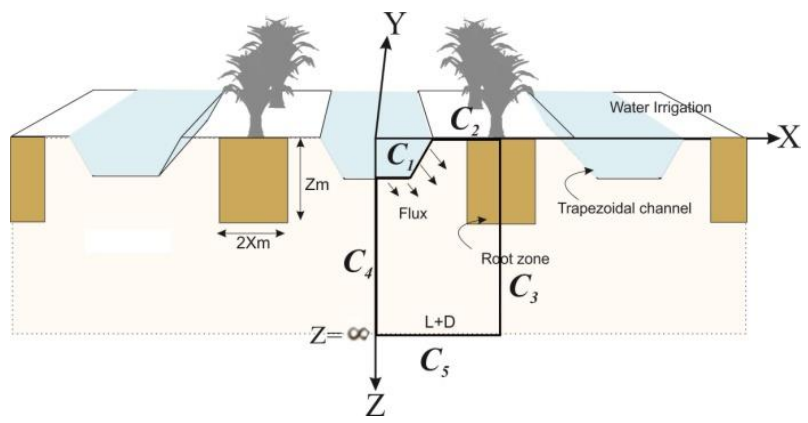

Figure 1 Geometry of periodic trapezoidal channels with the root water uptake.

\subsection{Basic Equation}

Model of water flow in unsaturated soils for unsteady infiltration with root water uptake is given by

$$
\begin{aligned}
& \frac{\partial \theta}{\partial T}=\frac{\partial}{\partial X}\left(K(\psi) \frac{\partial \psi}{\partial X}\right)+\frac{\partial}{\partial Z}\left(K(\psi) \frac{\partial \psi}{\partial Z}\right)- \\
& \frac{\partial K(\psi)}{\partial Z}-S(X, Z, \psi) .
\end{aligned}
$$

Where $\theta(-)$ is water content in the soil, $T(\mathrm{~T})$ is the time of infiltration, $K\left(\mathrm{LT}^{-1}\right)$ is the hydraulic conductivity, $\psi(\mathrm{L})$ is the suction potential, and $S\left(\mathrm{~T}^{-1}\right)$ is root water uptake function.

Model of root water uptake is defined as

$$
S(X, Z, \psi)=\gamma(\psi) \frac{L_{t} \beta(X, Z) T_{p o t}}{\int_{0}^{Z_{m}} \int_{L+D-X_{m}}^{L+D} \beta(X, Z) d X d Z}
$$

Here, $\gamma \in[0,1]$ is the water stress response function $L_{t}(\mathrm{~L})$ is soil surface width of transpiration process, $T_{p o t}$ $\left(\mathrm{LT}^{-1}\right)$ is potential transpiration, and $\beta(X, Z)\left(\mathrm{L}^{-2}\right)$ as the spatial root-water uptake distribution is formulated by

$$
\begin{aligned}
& \beta(X, Z)=\left(1-\frac{L+D-X}{X_{m}}\right)\left(1-\frac{Z}{Z_{m}}\right) \times \\
& \left(e^{-\left(\frac{p_{Z}}{Z_{m}}\left|Z^{*}-Z\right|+\frac{p_{X}}{X_{m}}\left|X^{*}-(L+D-X)\right|\right)}\right) .
\end{aligned}
$$

for $L+D-X_{m} \leq X \leq L+D$, and $0 \leq Z \leq Z_{m}$ where $p_{X}, p_{Z}, X^{*}, Z^{*}$ are fitting parameters.

The matrix flux potential or MFP $\left(\mathrm{L}^{2} \mathrm{~T}^{-1}\right)$ is obtained using Kirchhoff transformation defined by

$$
\Theta=\int_{-\infty}^{\psi} K(s) d s .
$$

Where $K(\psi)=K_{S} e^{\alpha \psi}, \alpha\left(\mathrm{L}^{-1}\right)$ is an empirical parameter and $K_{S}(\mathrm{LT}-1)$ is the saturated hydraulic conductivity.

As discussed by Solekhudin and Ang [6], For The Equation $\frac{\partial \theta}{\partial T}$ can be expressed as

$$
\frac{\partial \theta}{\partial T}=\frac{1}{D(\theta)} \frac{\partial \theta}{\partial T}
$$

Then, Equation (1) can be transformed to

$$
\begin{array}{r}
\frac{1}{D(\theta)} \frac{\partial \Theta}{\partial T}=\frac{\partial^{2} \Theta}{\partial X^{2}}+\frac{\partial^{2} \theta}{\partial Z^{2}}-\alpha \frac{\partial \theta}{\partial Z} \\
-S(X, Z, \psi) .
\end{array}
$$

The diffusivity may be assumed constant $d[6]$ and the suction potential is

$$
\psi=\frac{1}{\alpha} \ln \left(\frac{\theta \alpha}{K_{S}}\right) \text {. }
$$

Applying dimensionless variable

$$
\begin{aligned}
& \varphi(x, z, t)=\frac{\pi \theta}{v_{0} L}, \quad x=\frac{\alpha}{2} X, \quad z=\frac{\alpha}{2} Z, t= \\
& \frac{\alpha^{2} d}{4} T, \\
& u=\frac{2 \pi}{v_{0} \alpha L} U, v=\frac{2 \pi}{v_{0} \alpha L} V, \quad f=\frac{2 \pi}{v_{0} \alpha L} F
\end{aligned}
$$

and the transformation

$\varphi=\chi e^{z}$.

We obtain the equation as below

$$
\begin{aligned}
\frac{\partial \chi}{\partial t}=\frac{\partial^{2} \chi}{\partial x^{2}}+\frac{\partial^{2} \chi}{\partial z^{2}} & -\chi \\
& -\gamma^{*}(\chi) s_{m}^{*}(x, z) e^{-z}
\end{aligned}
$$

where

$$
\gamma^{*}(\chi)=\gamma\left(\frac{1}{\alpha} \ln \left(\frac{\alpha v_{0} L \chi e^{-z}}{\pi K_{S}}\right)\right)
$$

and $s_{m}{ }^{*}(x, z)$ is similar to Solekhuddin and Ang [8]

$$
S_{m}^{*}(x, z)=\frac{2 \pi}{\alpha L} \frac{l_{t} \beta^{*}(x, z)}{\int_{0}^{z_{m}} \int_{a}^{b} \beta^{*}(x, z) d x d z} \frac{T_{p o t}}{v_{0}}
$$

with

$\beta^{*}(x, z)=\left(1-\frac{b-x}{x_{m}}\right)\left(1-\frac{z}{z_{m}}\right) e^{-\frac{2}{\alpha}\left(\frac{p_{x}}{x_{m}}\left|x^{*}-(b-x)\right|+\frac{p_{z}}{z_{m}}\left|z^{*}-z\right|\right)}$

for $b-x_{m} \leq x \leq b$, and $0 \leq z \leq z_{m}$

here, $l_{t}=\frac{\alpha}{2} L_{t}, x^{*}=\frac{\alpha}{2} x^{*}, z^{*}=\frac{\alpha}{2} Z^{*}, x_{m}=\frac{\alpha}{2} X_{m}$,

$z_{m}=\frac{\alpha}{2} Z_{m}, p_{x}=\frac{\alpha}{2} P_{x}, p_{z}=\frac{\alpha}{2} P_{z}$ and $b=\frac{\alpha}{2}(L+D)$

Then, suction potential can be expressed ad

$\psi=\frac{1}{\alpha} \ln \left(\frac{\alpha v_{0} L \chi e^{-z}}{\pi K_{s}}\right)$

We first consider the nonlinear term $\gamma^{*}(\chi)$ as a constant. Making use of Laplace transform 


$$
\phi(x, z, s)=\int_{0}^{\infty} e^{-s t} \chi(x, z, t) d t
$$

with the initial condition

$$
\chi(x, z, 0)=0
$$

Equation (3) can be transformed into a modified Helmholtz equation as below

$$
\begin{aligned}
\frac{\partial^{2} \phi}{\partial x^{2}}+\frac{\partial^{2} \phi}{\partial z^{2}}=(1 & +s) \phi \\
& +\frac{e^{-z}}{s} \gamma^{*}(\chi) s_{m}{ }^{*}(x, z)
\end{aligned}
$$

Using the set of transformations above, the boundary conditions for unsteady infiltration with root water uptake are expressed by

$$
\begin{array}{llrl}
\frac{\partial \phi}{\partial n} & =\phi n_{2}+\frac{2 \pi}{\alpha L s} e^{-z} & & \mathrm{C}_{1}, \\
\frac{\partial \phi}{\partial n}=-\phi & \mathrm{C}_{2} \text { and } \mathrm{C}_{5}, \\
\frac{\partial \phi}{\partial n}=0 & \mathrm{C}_{3} \text { and } \mathrm{C}_{4} .
\end{array}
$$

The term $n_{2}$ in boundary condition Equation (5) is the vertical component normal vector region $R$.

\subsection{The DRM with A Predictor-Corrector Scheme}

The integro-differential equation of the modified Helmholtz Equation (4) and the boundary conditions Equation (5)-(7) is

$$
\begin{aligned}
& \lambda(\xi, \eta) \phi(\xi, \eta, s)= \\
& -\int_{C}\left(\phi(x, z, s) \frac{\partial \Upsilon(x, z ; \zeta, \zeta)}{\partial n}-\right. \\
& \left.\Upsilon(x, z ; s, \zeta) \frac{\partial \phi(x, z, s)}{\partial n}\right) d s(x, z)+ \\
& \iint_{R} \Upsilon(x, z ; \varsigma, \zeta)\left(\frac{e^{-z}}{s} \gamma^{*}(\chi) s_{m}{ }^{*}(x, z)+(1+\right. \\
& s) \phi(x, z, s)) d x d z .
\end{aligned}
$$

Where $Y(x, z ; \varsigma, \zeta)$ is the fundamental solution of twodimensional Laplace equation and

$$
\lambda(\xi, \eta)=\left\{\begin{array}{cc}
\frac{1}{2} & (\xi, \eta) \text { on smooth part of } C \\
1 & (\xi, \eta) \text { on domain } R .
\end{array}\right.
$$

Equation (8) can be solved numerically using DRM and a predictor-corrector scheme simultaneously. The function $\gamma^{*}(\chi)$ is determined by using a predictorcorrector scheme. The procedure can be referred to [9] for the detailed method. The process will be stopped until

$$
\sum_{i=1}^{N+M}\left|\gamma_{j}^{*(i)}-\gamma_{j-1}^{*(i)}\right|=\delta<\varepsilon
$$

to acquire the convergence condition.

The numerical results of this problem are obtained in the Laplace domain. Then, the dimension MFP numerical value is determined by employing The Stehfest formulae as inverse of Laplace transform. The formulae are

$$
\chi(x, z, t) \cong \frac{\log (2)}{t} \sum_{p=1}^{2 P} K_{p} \phi(x, z, s) .
$$

Where

$$
\begin{aligned}
& s_{p}=p \frac{\log (2)}{t}, K_{p}=(-1)^{(p+P)} \times \\
& \sum_{m=\frac{\cot }{2}}^{\min (p, P)} \frac{m^{2}(2 m) !}{(P-m) ! m !(m-1) !(p-m) !(2 m-p) !} \\
& p, P \text { are positive integers, } P=\frac{1}{2} p
\end{aligned}
$$

Based on dimensionless variable $t=$ $\frac{\alpha^{2} d}{4} T$ and $d$ is water diffusivity factor of soils.

\section{RESULT AND DISCUSSION}

This section describes the numerical result through a problem of root water uptake process for different types of homogeneous soil in unsteady infiltration. The explained methods in the previous section evaluate the unsteady infiltration for clay-loam, clay, and silt-loam homogenous soils. The hydraulic properties of soils based on Warrick's study [10] and the soil water diffusivity according to Chaudhari and Somawanshi [5] are presented in Table 1.

Table 1. Values $\alpha$, Ks and D

\begin{tabular}{lccc}
\hline & $\alpha\left(\mathrm{cm}^{-1}\right)$ & $\mathrm{Ks}(\mathrm{cm} /$ day $)$ & $\mathrm{D}\left(\mathrm{cm}^{2} \mathrm{~min}^{-1}\right)$ \\
\hline clay-loam & $1.9 \times 10^{-2}$ & 6.24 & $3,3 \times 10^{-6} e^{36.4 \theta v}$ \\
\hline silt-loam & $2 \times 10^{-2}$ & 10.8 & $4.8 \times 10^{-2} e^{21.9 \theta v}$ \\
\hline clay & $8 \times 10^{-3}$ & 4.8 & $4.39 \times 10^{-8} e^{38.3 \theta v}$ \\
\hline
\end{tabular}

Here, $\theta v$ is the saturated volumetric water content of soils. the values are 0.5 for clay-loam, 0.4 for silt-loam and 0.6 for clay. Corresponding values of TEC and SAR to determine $D(\theta)$ are $5 \mathrm{meq}^{-1}$ and $5 \mathrm{~m} \mathrm{~mol}^{1 / 2} \mathrm{l}^{-1 / 2}$, respectively.

For the root zone of crops, we set $L t=X m=$ $50 \mathrm{~cm}$ and $\mathrm{Zm}=100 \mathrm{~cm}$ as reported by Vrugt [9]. The fitted parameters of the root zone are $X^{*}=0 \mathrm{~cm}, P x=$ $1.00, Z^{*}=20 \mathrm{~cm}$, and $P z=1.00$. Potential transpiration in this study is set to be $0.4 \mathrm{~cm} /$ day The relation between root-water stress response function, $\gamma$ and suction potential, $\psi$, is the same as that reported by Utset et al [11].

The Laplace Transform of DRM with a predictorcorrector needs discretization for boundary $(N)$ and interior points $(M)$. After performing several computational experiments, we set $\mathrm{N}=407, \mathrm{M}=899$ for Clay, $\mathrm{N}=401, \mathrm{M}=892$ for Clay-Loam, and Silt-Loam.

\subsection{MFP and Root-Water Uptake Process}

The numerical results of the water absorption effect by crop roots are obtained by comparing the MFP value from the corresponding problem with and without rootwater uptake process. The MFP are evaluated from time 
to time until reaching the steady condition for Clay-Loam Silt-Loam and Clay Soils. The results obtained are presented in Figure 2- Figure 4

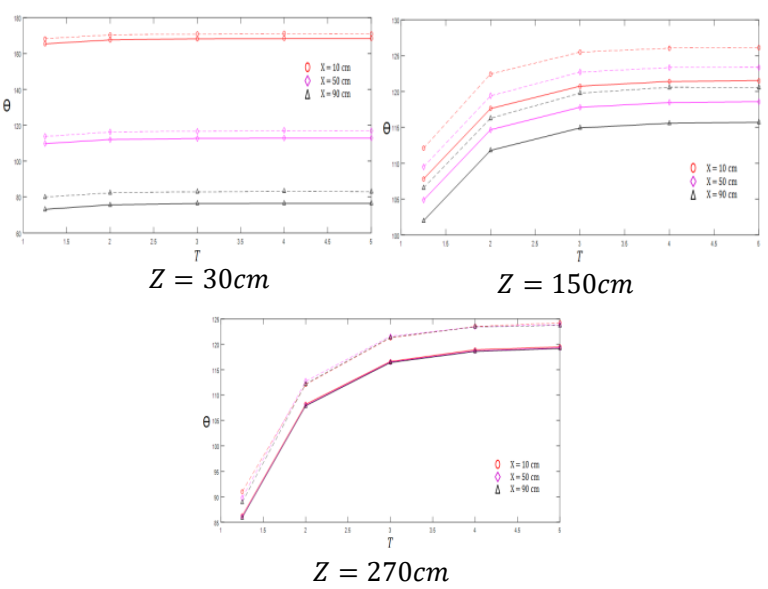

Figure 2 Variation of MFP with T for infiltration with root-water uptake (solid lines), and without root-water uptake (dashed lines) in Clay-Loam soil at three locations along Z-axis.

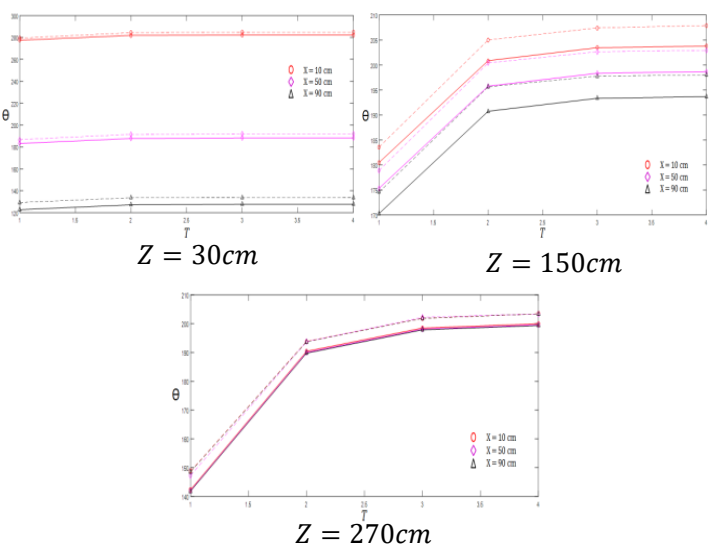

Figure 3 Variation of MFP with T for infiltration with root-water uptake (solid lines), and without root-water uptake (dashed lines) in Silt-Loam soil at three locations along Z-axis.

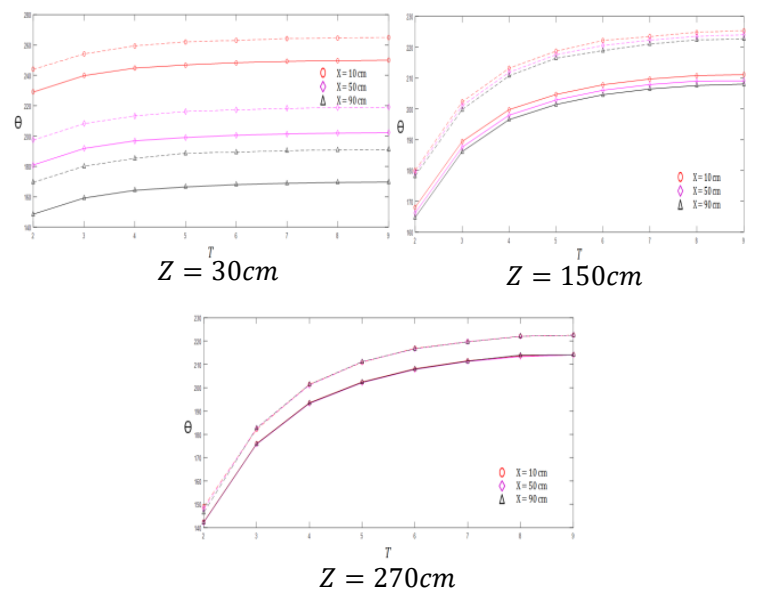

Figure 4 Variation of MFP with T for infiltration with root-water uptake (solid lines), and without root-water uptake (dashed lines) in Clay soil at three locations along Z-axis.

The Figure 2 - Figure 4 show the variation of MFP as $\mathrm{T}$ increases in Clay-Loam, Silt-Loam, and Clay soils, for infiltration with and without root-water uptake along with three different locations, $Z=30 \mathrm{~cm}, \quad Z=150 \mathrm{~cm}$ and $Z=270 \mathrm{~cm}$, respectively. For each horizontal location, three points are selected such one point equidistant from two boundaries $(X=0 \mathrm{~cm}$ and $X=$ $100 \mathrm{~cm}$ ), at $X=50 \mathrm{~cm}$, and the others near to the boundary, at $X=10 \mathrm{~cm}$ and $X=90 \mathrm{~cm}$.

These figures present the difference in MFP between infiltration with and without root-water uptake increases as $\mathrm{X}$ increases. It describes when the distance from the plant decreases, the difference in MFP rises. This implies that the water-uptake process of crop roots at a point near the plant is higher than those at points further. In other words, the maximum uptake is the nearest to the plant.

Comparing the infiltration process between three homogeneous soils in Figure 2 - Figure 4, It can be seen that the difference MFP values between infiltration with and without root-water uptake the highest to the lowest respectively are in Figure 4, Figure 2, and Figure 3. In other words, the root water uptake process or the total amount of water absorbed among the three homogeneous soil, Clay gives the highest and Silt-Loam gives the lowest. Based on the hydraulic properties of soils, The empirical parameter of soil, $\alpha$, indicates the coarseness texture of the soil. The greater value of $\alpha$ gives the more coarseness texture of soil. As given in Tabel 1, These results imply that the root-water uptake process in finer soil types provide higher amount of water absorbed than those coarser.

\subsection{Distribution of Root Water Uptake in The Root Zone}

The estimation of distribution root water uptake is evaluated to obtain the amount of water absorbed for each zone by crop roots in three types of homogeneous soils. To estimate the actual amount of water absorbed by crops, parameters are transformed back using the dimensionless variables. Then, Equation (2) as the sink term of Equation (1) is used to determine the $S$ value.

In this study, the root zone is defined by

$\{(X, Z): 50 \mathrm{~cm} \leq X \leq 100 \mathrm{~cm}$ and $0 \leq Z \leq 100 \mathrm{~cm}\}$.
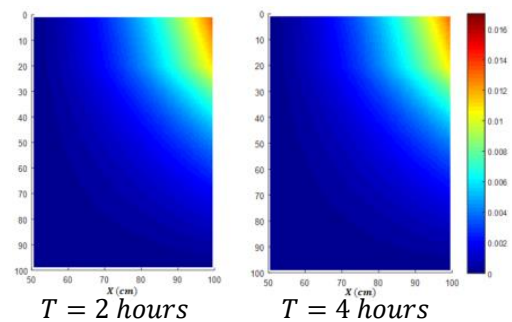
Figure 5 Surface plot of root uptake function for ClayLoam soil at two times.

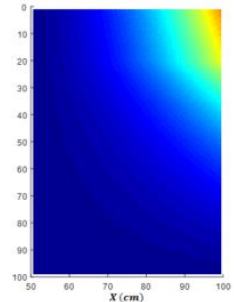

$T=2$ hours

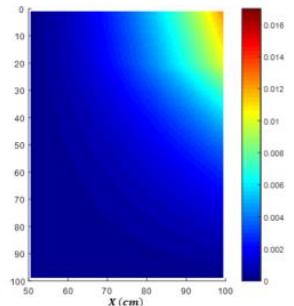

$T=4$ hours
Figure 6 Surface plot of root uptake function for SiltLoam soil at two times.
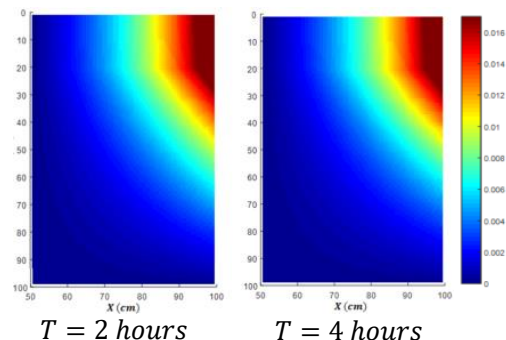

Figure 7 Surface plot of root uptake function for Clay soil at two times.

Figure 5- Figure 7 visualize the root water uptake in two similar times of unsteady infiltration in order to compare the water distribution among Clay-Loam, SiltLoam, and Clay soils. From the figures, it can be seen that the maximum uptake occurs at the point about $X=100$ $\mathrm{cm}$ from the line $Z=0 \mathrm{~cm}$ until $Z=20 \mathrm{~cm}$ from the surface of the soil. These results are suitable to fitting parameters of root-uptake function according to $X^{*}=$ $0 \mathrm{~cm}$, and $Z^{*}=20 \mathrm{~cm}$. It can also be seen that value of $\mathrm{S}$ decrease as $\mathrm{T}$ increases, at any point in the root zone.

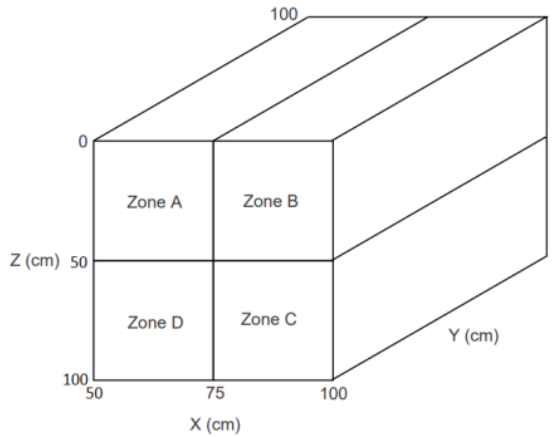

Figure 8 Four zones of root water uptake.

To examine the difference in the total amount of water absorbed between such regions quantitatively, this root zone with a length of $100 \mathrm{~cm}$ along OY direction is considered and then divided into four zones, namely Zone A, Zone B, Zone C, and Zone D which illustrated by Figure 8
Zone $\mathrm{A}=\{(X, Y, Z): 50 \mathrm{~cm} \leq X \leq 75 \mathrm{~cm}, 0 \leq$ $Y \leq 100 \mathrm{~cm}$, and $0 \leq Z \leq 50 \mathrm{~cm}\}$,

Zone $\mathrm{B}=\{(X, Y, Z): 75 \mathrm{~cm} \leq X \leq 100 \mathrm{~cm}, 0 \leq$ $Y \leq 100 \mathrm{~cm}$, and $0 \leq Z \leq 50 \mathrm{~cm}\}$,

Zone $\mathrm{C}=\{(X, Y, Z): 75 \mathrm{~cm} \leq X \leq 100 \mathrm{~cm}, 0 \leq$ $Y \leq 100 \mathrm{~cm}$, and $50 \mathrm{~cm} \leq Z \leq 100 \mathrm{~cm}\}$,

Zone $\mathrm{D}=\{(X, Y, Z): 50 \mathrm{~cm} \leq X \leq 75 \mathrm{~cm}, 0 \leq$ $Y \leq 100 \mathrm{~cm}$, and $50 \mathrm{~cm} \leq Z \leq 100 \mathrm{~cm}\}$.

The formulae used to compute the total amount of water absorbed from Zone A, Zone B, Zone C, and Zone D, are

$$
\begin{aligned}
& 100 \times \int_{0}^{50} \int_{50}^{75} S(X, Z, \Psi) d X d Z, \\
& 100 \times \int_{0}^{50} \int_{75}^{100} S(X, Z, \Psi) d X d Z, \\
& 100 \times \int_{50}^{100} \int_{100}^{100} S(X, Z, \Psi) d X d Z, \\
& 100 \times \int_{50}^{75} \int_{50}^{75} S(X, Z, \Psi) d X d Z .
\end{aligned}
$$

The function $\mathrm{S}$ can only be estimated numerically because the water-stress response function, $\gamma(\psi)$, may not be determined analytically. Thus, each zone is divided into $50 \times 50$ rectangular region with breadth $\Delta \mathrm{x}$ and length $\Delta z$, to compute Equation (9)-(12) numerically. Hence, Equation (9)-(12) may be approximated by

$$
100 \times \sum_{j=1}^{50} \sum_{i=1}^{50} S_{i j} \Delta x_{i} \Delta z_{j}
$$

where $S_{i j}$ is the value of $\mathrm{S}$ at one corner of the rectangular region at $\mathrm{i}$-th row and $\mathrm{j}$-th column, and $\Delta x_{i}=\Delta x$ and $\Delta z_{j}=\Delta z$ are the breadth and the length of this rectangular regions.

Employing Equation (13), the numerical results of the water uptake or the total amount water absorbed by crop roots at 2 hours and 4 hours are given in Table 2. 
Table 2. Root water uptake at different zone in three different types of homogeneous soils

\begin{tabular}{ccccccc}
\hline \multicolumn{7}{c}{ The total amount of water absorbed by crop } \\
roots $\left(\mathrm{cm}^{3} /\right.$ day) \\
\cline { 2 - 7 } $\mathrm{t}$ (time) & ZoneA & ZoneB & ZoneC & ZoneD & Total \\
\hline \multirow{2}{*}{$\mathrm{CL}$} & 2 & 131.73 & 676.35 & 129.06 & 26.50 & 963.64 \\
\cline { 2 - 7 } & 4 & 130.67 & 670.29 & 127.45 & 26.2 & 954.61 \\
\hline \multirow{2}{*}{$\mathrm{SL}$} & 2 & 123.78 & 634.42 & 119.98 & 24.63 & 902.80 \\
\cline { 2 - 7 } & 4 & 123.33 & 631.91 & 119.30 & 24.50 & 899.04 \\
\hline \multirow{2}{*}{$\mathrm{C}$} & 2 & 174.29 & 790.72 & 517.96 & 113.19 & 1596.15 \\
\cline { 2 - 7 } & 4 & 172.4 & 790.71 & 509.23 & 106.98 & 1579.32 \\
\hline
\end{tabular}

Table 2 shows the root water uptake from Zone B is much higher than those from other zones. Visually, it can be seen from Figure 6 - Figure 8. Besides that, the amount of water absorbed by crop roots decreases as $T$ increases.

The total amount of water absorbed by crop roots from Clay-Loam, Silt-Loam, and Clay soils at 2 hours over are $963.64 \mathrm{~cm}^{3} /$ day, $902.80 \mathrm{~cm}^{3} /$ day, 1579.32 $\mathrm{cm}^{3} /$ day, and at 4 hours are $954.61 \mathrm{~cm}^{3} /$ day, $899.04 \mathrm{~cm}^{3} /$ day, $1579.32 \mathrm{~cm}^{3} /$ day. In other words, The total amount of root water uptake from higher to lower respectively are Clay $>$ Clay - Loam $>$ Silt - Loam. As expected from the previous discussion, these numerical values indicate that crops absorb more water from finer soils than coarser soils.

\section{CONCLUSION}

A problem of root water uptake process from different types of homogeneous soil in unsteady infiltration has been solved numerically. The numerical method are dual reciprocity method with a predictor-corrector scheme in the Laplace domain. After the governing equation is solved numerically, Stehfest formulae and diffusivity factor are employed to obtain MFP in actual time until going steady. Finally, MFP are used to estimate the root water uptake or the water absorption by crop roots in two times to compare the numerical result between three types of homogeneous soils.

The results indicate that the water-uptake process of crop roots at a point near the plant is higher than those at points further. In other words, the maximum uptake is the nearest to the plant. From a time-dependent perspective, the amount of water absorbed by crop roots decreases as time increases. Besides that, these results also imply that the root-water uptake process in finer soil types provide higher amount of water absorbed than those coarser.

\section{ACKNOWLEDGMENTS}

The first author wishes to thank the Faculty of Mathematics and Natural Science, Universitas Jember for providing financial support for this research

\section{REFERENCES}

[1] H.A. Basha, Multidimensional linearized nonsteady infiltration with prescribed boundary conditions at the soil surface, Water Resources Research 35, 1999, pp. 75-83, DOI: 10.1029/1998 WR900015

[2] D.A. Barry, J.Y. Parlange, Infiltration subject to time-dependent surface ponding: exact results and correspondence to solute transport with nonlinear reaction, Subsurface-Water Hydrology, 1996, pp. 33-48, DOI: 10.1007/978-94-011-0391-6_3

[3] D.L. Clements, M. Lobo, A BEM for time dependent infiltration from an irrigation channel, Engineering Analysis with Boundary Elements 34, 2010, pp. 1100-1104, DOI: $\quad 10.1016 /$ j.enganabound. 2010.06.013

[4] M. Zahroh, I. Solekhudin, Steady state conditions predictions of time-dependent infiltration problems: an LTDRM with a predictor-corrector scheme approach, Journal of Physics: Conference Series, Volume 1280, Issue 2, 2019, DOI: :10.1088/1742-6596/1280/2/022039

[5]. S.K. Chaudhari, R.B. Somawanshi, Unsaturated flow of fifferent quality irrigation waters through Clay, Clay Loam and Silt Loam soils and its dependence on soil and solution parameters, Agricultural Water Management 64, 2004, pp. 6990. DOI: 10.1016/S0378-3774(03)00145-8

[6] I. Solekhudin, K.C. Ang, A Laplace transform DRBEM with a predictor-corrector scheme for time-dependent infiltration from periodic channels with root-water uptake, Engineering Analysis with Boundary Elements 50, 2015, pp. 141-147, DOI: 10.1016/j.enganabound.2014.08.011

[7] V. Batu, Steady infiltration from single and periodic strip sources, Soil Science Society America Journal 42, 1978, pp. 544-549, DOI: 10.2136/sssaj1978 $03615995004200040002 x$

[8] I. Solekhudin, K.C. Ang, A DRBEM with a predictor-corrector scheme for steady infiltration from periodic channels with root-water uptake. Engineering Analysis with Boundary Elements 36, 2012, pp. 1199-204, DOI: 10.1016/j.enganabound .2012 .02 .013 
[9] J.A.Vrugt, J.W. Hopmans, Simunek J. Calibration of a two-dimensional root "water uptake model. Soil Science Society American Journal, 65, 2001, pp.1027-37.DOI: 10.2136/sssaj2001.6541027x

[10] A.W. Warrick, Soil Physics Companion, Washington DC: CRC Press, 2002.
[11] A. Utset, M.E. Ruiz, J. Garcia and R.A. Feddes, A SWACROP-based potato root water-uptake function as determined under tropical conditions, Potato Research 43, 2000, pp. 19-29, DOI: 10.1007/BF02358510 